\title{
A New Generalization of the Lomax Distribution with Increasing, Decreasing, and Constant Failure Rate
}

\author{
Pelumi E. Oguntunde, ${ }^{1}$ Mundher A. Khaleel, ${ }^{2}$ Mohammed T. Ahmed, ${ }^{3}$ \\ Adebowale O. Adejumo, ${ }^{1,4}$ and Oluwole A. Odetunmibi ${ }^{1}$ \\ ${ }^{1}$ Department of Mathematics, Covenant University, Ota, Ogun State, Nigeria \\ ${ }^{2}$ Department of Mathematics, Faculty of Computer Science and Mathematics, University of Tikrit, Tikrit, Iraq \\ ${ }^{3}$ Department of Finance and Banking, University of Tikrit, Tikrit, Iraq \\ ${ }^{4}$ Department of Statistics, University of Ilorin, Ilorin, Kwara State, Nigeria
}

Correspondence should be addressed to Pelumi E. Oguntunde; peluemman@yahoo.com

Received 20 April 2017; Accepted 1 June 2017; Published 19 July 2017

Academic Editor: Ricardo Perera

Copyright (C) 2017 Pelumi E. Oguntunde et al. This is an open access article distributed under the Creative Commons Attribution License, which permits unrestricted use, distribution, and reproduction in any medium, provided the original work is properly cited.

Developing new compound distributions which are more flexible than the existing distributions have become the new trend in distribution theory. In this present study, the Lomax distribution was extended using the Gompertz family of distribution, its resulting densities and statistical properties were carefully derived, and the method of maximum likelihood estimation was proposed in estimating the model parameters. A simulation study to assess the performance of the parameters of Gompertz Lomax distribution was provided and an application to real life data was provided to assess the potentials of the newly derived distribution. Excerpt from the analysis indicates that the Gompertz Lomax distribution performed better than the Beta Lomax distribution, Weibull Lomax distribution, and Kumaraswamy Lomax distribution.

\section{Introduction}

The Lomax distribution can also be called Pareto Type II distribution and its application can be found in many fields like actuarial science, economics, and so on [1]. The distribution was defined by Lomax [2] and it is a heavy-tailed distribution. It has also been considered to be useful in reliability and life testing problems in engineering and in survival analysis as an alternative distribution $[3,4]$.

Modified and extended versions of the Lomax distribution have been studied; examples include the weighted Lomax distribution [4], exponential Lomax distribution [5], exponentiated Lomax distribution [6], gamma Lomax distribution [7], transmuted Lomax distribution [8], Poisson Lomax distribution [9], McDonald Lomax distribution [10], Weibull Lomax distribution [11], and power Lomax distribution [12]. Besides, estimation of the parameters of Lomax distribution under general progressive censoring has also been considered by Al-Zahrani and Al-Sobhi [1].
In addition to the generalized families of distributions mentioned earlier, there are several other generalized families of distributions in the literature and these are contained in Owoloko et al. [13], Oguntunde et al. [14], Cordeiro et al. [15], and Alizadeh et al. [16]. Meanwhile, of interest to us in this research is to extend the Lomax distribution using the Gompertz generalized family of distributions due to Alizadeh et al. [16] because it is relatively new; it has not yet been rigorously explored, and it has some potentials which would be revealed in the later part of this article.

The rest of this article is therefore organized as follows: in Section 2, the densities of the Gompertz Lomax distribution (henceforth, it is referred to as GoLom distribution) are derived; its statistical properties are established including estimation of the unknown parameters; in Section 3, a simulation study was provided to investigate the performances of the unknown parameters; then an application to a real life data was provided, followed by a concluding remark. 


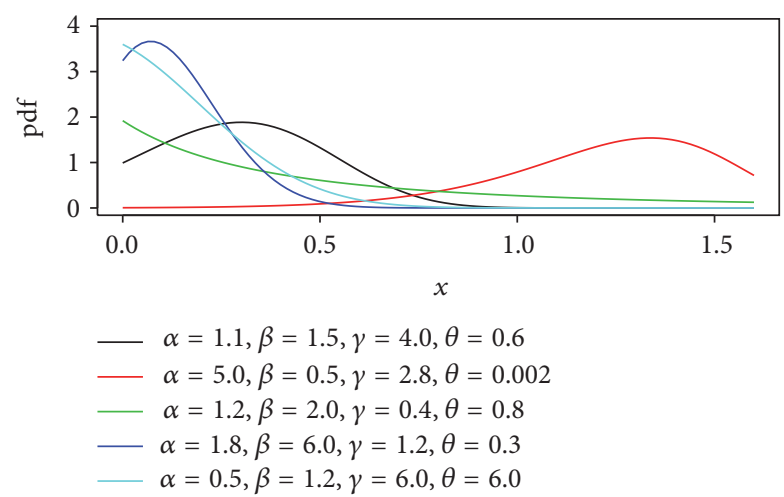

Figure 1: Plot for the pdf of GoLom distribution.

\section{The Gompertz Lomax Distribution}

To start with, the cumulative distribution function (cdf) and probability density function (pdf) of the Lomax distribution with parameters $\alpha$ and $\beta$ are given by

$$
\begin{aligned}
& G(x)=\left[1-(1+\beta x)^{-\alpha}\right] ; \quad \alpha>0, \beta>0, \\
& g(x)=\alpha \beta(1+\beta x)^{-(\alpha+1)} ; \quad \alpha>0, \beta>0,
\end{aligned}
$$

respectively, where $\alpha$ and $\beta$ are the shape and scale parameters, respectively.

According to Alizadeh et al. [16], the cdf and pdf of the Gompertz generalized family of distribution are given by

$$
\begin{gathered}
F(x)=1-e^{(\theta / \gamma)\left\{1-[1-G(x)]^{-\gamma}\right\}} ; \quad \theta>0, \quad \gamma>0, \\
f(x)=\theta g(x)[1-G(x)]^{-\gamma-1} e^{(\theta / \gamma)\left\{1-[1-G(x)]^{-\gamma}\right\}} ; \\
\theta>0, \quad \gamma>0,
\end{gathered}
$$

where $\theta$ and $\gamma$ are additional shape parameters and their role is to vary tail weights.

$G(x)$ and $g(x)$ are the cdf and pdf of the parent (or baseline) distribution, respectively.

Now, if the density in (1) in inserted into (3), then the cdf of the GoLom distribution is given by

$$
\begin{aligned}
& F(x)=1-e^{(\theta / \gamma)\left\{1-[1+\beta x]^{\alpha \gamma}\right\}} ; \\
& \theta>0, \gamma>0, \alpha>0, \beta>0 .
\end{aligned}
$$

Its associated pdf is derived by inserting the densities in (1) and (2) into (4) as follows:

$$
\begin{aligned}
& f(x)=\theta \alpha \beta(1+\beta x)^{\alpha \gamma-1} e^{(\theta / \gamma)\left\{1-[1+\beta x]^{\alpha \gamma}\right\}} ; \\
& \theta>0, \gamma>0, \alpha>0, \beta>0,
\end{aligned}
$$

where $\alpha, \theta$, and $\gamma$ are shape parameters; $\beta$ is the scale parameter.

Plots for the pdf of the GoLom distribution at various selected values are displayed in Figure 1.
Remark 1. It is clear in Figure 1 that the shape of the GoLom distribution could be decreasing or inverted bathtub (depending on the value of the parameters). Also, it could be positively skewed or negatively skewed. Studying the tail behaviour of the GoLom distribution, it can be deduced that the distribution is heavy-tailed.

2.1. Expansion for the Densities of GoLom Distribution. Following Alizadeh et al. [16], the density of the GoLom distribution in (5) can be expanded as follows:

$$
F(x)=1-\sum_{i=0}^{\infty} \sum_{j=0}^{i} \sum_{k=0}^{\infty} w_{i, j, k} H_{k}(x)=1-\sum_{k=0}^{\infty} a_{k} H_{k}(x),
$$

where $w_{i, j, k}=\left((-1)^{i+k} / i !\right)\left(\begin{array}{c}i \\ j\end{array}\right)\left(\begin{array}{c}-j \gamma \\ k\end{array}\right)(\theta / \gamma)^{i}, a_{k}=$ $\sum_{i=0}^{\infty} \sum_{j=0}^{i} w_{i, j, k}$, and $H_{k}(x)$ is the cdf of the exponentiated Lomax distribution with power $k>0$.

The associated pdf can be expressed as a linear mixture of the exponentiated Lomax function as follows:

$$
f(x)=\sum_{k=0}^{\infty} b_{k+1} h_{k+1}(x),
$$

where $h_{k+1}=(k+1) g(x) G(x)^{k}$ and $b_{k}=-a_{k}$ and $G(x)$ and $g(x)$ are the cdf and pdf of the Lomax distribution as defined in (1) and (2), respectively.

2.2. Reliability Analysis. The expressions for the reliability function, hazard function (or failure rate), reversed hazard function, and odds function are all derived and established in this subsection.

Reliability Function. Reliability or survival function can be obtained from

$$
S(x)=1-F(x) .
$$

Therefore, the reliability function of the GoLom distribution is given by

$$
S(x)=e^{(\theta / \gamma)\left\{1-[1+\beta x]^{\alpha \gamma}\right\}} ;
$$

$$
\theta>0, \gamma>0, \alpha>0, \beta>0 .
$$

It is good to note that the shape of the reliability function of GoLom distribution would be a constant when the value of parameter $\beta=0$ and $\alpha=\theta=\gamma=1$. An illustration to this is as shown in Figure 2.

Hazard Function. Hazard function can be obtained from

$$
h(x)=\frac{f(x)}{S(x)} .
$$

Therefore, the hazard function of the GoLom distribution is given by

$$
\begin{aligned}
& h(x)=\theta \alpha \beta(1+\beta x)^{\alpha \gamma-1} ; \\
& \theta>0, \gamma>0, \alpha>0, \beta>0 .
\end{aligned}
$$

Plots for the hazard function of the GoLom distribution at various selected values are displayed in Figure 3. 


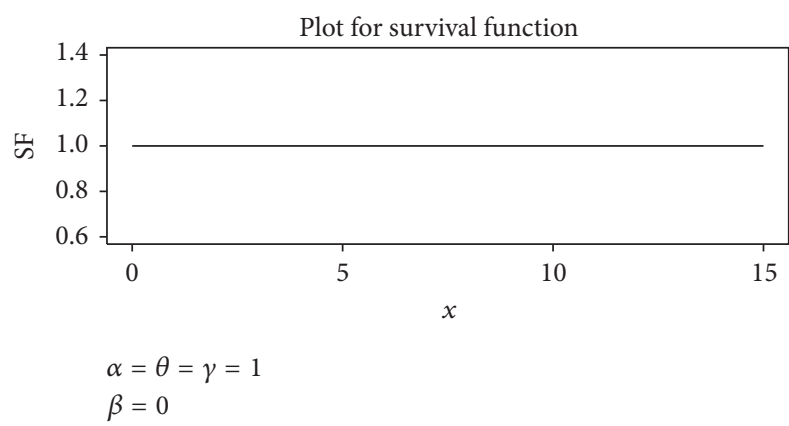

Figure 2: Survival function of GoLom distribution at $\beta=0$ and $\alpha=\theta=\gamma=1$.

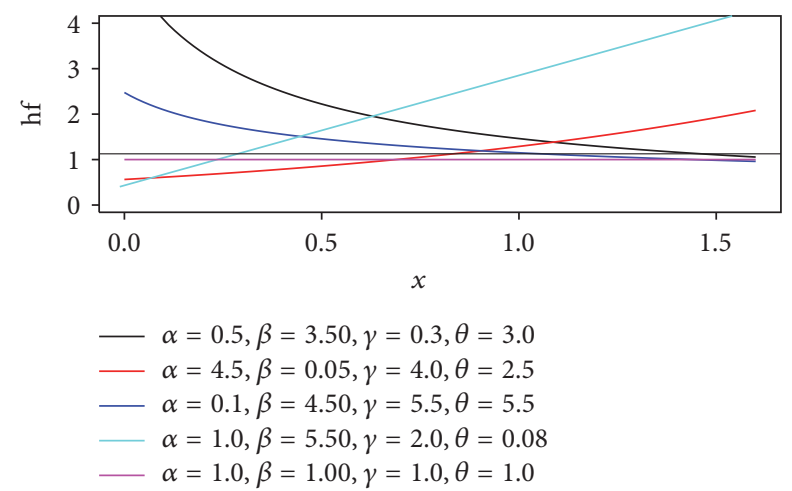

FIgURE 3: Plot for the hazard function of GoLom distribution.

Remark 2. It can be deduced from Figure 3 that the shape of the hazard function of the GoLom distribution could be constant, increasing, or decreasing (depending on the value of the parameters).

Reversed Hazard Function. Reversed hazard function can be derived from

$$
r(x)=\frac{f(x)}{F(x)} .
$$

Therefore, the reversed hazard function for the GoLom distribution is given by

$$
\begin{aligned}
& r(x)=\frac{\theta \alpha \beta(1+\beta x)^{\alpha \gamma-1} e^{(\theta / \gamma)\left\{1-[1+\beta x]^{\alpha \gamma}\right\}}}{1-e^{(\theta / \gamma)\left\{1-[1+\beta x]^{\alpha \gamma}\right\}}} \\
& \theta>0, \gamma>0, \alpha>0, \beta>0 .
\end{aligned}
$$

Odds Function. Odds function can be derived from

$$
O(x)=\frac{F(x)}{S(x)} .
$$

Therefore, the odds function for the GoLom distribution is given by

$$
\begin{aligned}
& O(x)=\frac{1-e^{(\theta / \gamma)\left\{1-[1+\beta x]^{\alpha \gamma}\right\}}}{e^{(\theta / \gamma)\left\{1-[1+\beta x]^{\alpha}\right\}}} ; \\
& \quad \theta>0, \gamma>0, \alpha>0, \beta>0 .
\end{aligned}
$$

2.3. Quantile Function and Median. Quantile function can be derived from

$$
Q(u)=F^{-1}(u) .
$$

Therefore, the quantile function of the GoLom distribution is given by

$$
Q(u)=\beta^{-1}\left\{\left[1-\frac{\gamma}{\theta} \log (1-u)\right]^{1 / \alpha \gamma}-1\right\},
$$

where $u \sim \operatorname{Uniform}(0,1)$.

Random numbers can be generated from the GoLom distribution using

$$
x=\beta^{-1}\left\{\left[1-\frac{\gamma}{\theta} \log (1-u)\right]^{1 / \alpha \gamma}-1\right\} .
$$

The median of the GoLom distribution can be derived by substituting $u=0.5$ into (18) as follows:

$$
\text { Median }=\beta^{-1}\left\{\left[1-\frac{\gamma}{\theta} \log (0.5)\right]^{1 / \alpha \gamma}-1\right\} .
$$

Other quartiles can also be derived from (18) by substituting the appropriate value of " $u$."

2.4. Distribution of Order Statistics. Let $x_{1}, x_{2}, \ldots, x_{n}$ be a random sample from a cdf and pdf of a Gompertz Lomax distribution as defined in (5) and (6), respectively; the pdf of the $j$ th order statistics of the GoLom distribution is obtained from

$$
\begin{aligned}
& f_{j: n}(x) \\
& \quad=\frac{n !}{(j-1) !(n-j) !} f(x) F(x)^{j-1}[1-F(x)]^{n-j} .
\end{aligned}
$$

Then, the pdf of $j$ th order statistics for the GoLom distribution is

$$
\begin{gathered}
f_{j: n}(x)=\frac{n !}{(j-1) !(n-j) !} \theta \alpha \beta(1+\beta x)^{\alpha \gamma-1} \\
\cdot e^{(\theta / \gamma)\left\{1-[1+\beta x]^{\alpha \gamma}\right\}}\left[1-e^{(\theta / \gamma)\left\{1-[1+\beta x]^{\alpha \gamma}\right\}}\right]^{j-1} \\
\cdot\left[e^{(\theta / \gamma)\left\{1-[1+\beta x]^{\alpha \gamma}\right\}}\right]^{n-j} .
\end{gathered}
$$

Therefore, the distribution of minimum and maximum order statistics for the GoLom distribution is given by

$$
\begin{aligned}
& f_{1: n}(x)=n \theta \alpha \beta(1+\beta x)^{\alpha \gamma-1} \\
& \cdot e^{(\theta / \gamma)\left\{1-[1+\beta x]^{\alpha \gamma}\right\}}\left[e^{(\theta / \gamma)\left\{1-[1+\beta x]^{\alpha \gamma}\right\}}\right]^{n-1}, \\
& f_{n: n}(x)=n \theta \alpha \beta(1+\beta x)^{\alpha \gamma-1} \\
& \cdot e^{(\theta / \gamma)\left\{1-[1+\beta x]^{\alpha \gamma}\right\}}\left[1-e^{(\theta / \gamma)\left\{1-[1+\beta x]^{\alpha \gamma}\right\}}\right]^{n-1} .
\end{aligned}
$$


2.5. Parameter Estimation. The parameters of the GoLom distribution can be estimated using the method of maximum likelihood (MLE) as follows: let $x_{1}, x_{2}, \ldots, x_{n}$ denote random samples each having the pdf of the GoLom distribution; then the likelihood function is given by

$$
\begin{aligned}
f & \left(x_{1}, x_{2}, \ldots, x_{n} ; \alpha, \beta, \gamma, \theta\right) \\
& =\prod_{i=1}^{n}\left[\theta \alpha \beta\left(1+\beta x_{i}\right)^{\alpha \gamma-1} e^{(\theta / \gamma)\left\{1-\left[1+\beta x_{i}\right]^{\alpha \gamma}\right\}}\right] .
\end{aligned}
$$

Let $l$ denote the log-likelihood function; that is, let $l=$ $\log f\left(x_{1}, x_{2}, \ldots, x_{n} ; \alpha, \beta, \gamma, \theta\right)$; then

$$
\begin{gathered}
l=n \log (\theta)+n \log (\alpha)+n \log (\beta) \\
+(\alpha \gamma-1) \sum_{i=1}^{n} \log \left(1+\beta x_{i}\right) \\
+\frac{\theta}{\gamma} \sum_{i=1}^{n}\left\{1-\left(1+\beta x_{i}\right)^{\alpha \gamma}\right\} .
\end{gathered}
$$

Solving $d l / d \alpha=0, d l / d \beta=0, d l / d \gamma=0$, and $d l / d \theta=0$ simultaneously gives the maximum likelihood estimates of parameters $\alpha, \beta, \gamma$, and $\theta$. Meanwhile, the solution cannot be gotten analytically except numerically when data sets are available. Software like R, MATLAB, MAPLE, and so on could be used to get the estimates.

\section{Simulation}

The behaviour of the parameters of the GoLom distribution was investigated by conducting simulation studies with the aid of R software. Data sets were generated from the GoLom distribution with a replication number $m=1000$; random samples of sizes $n=25,50$, and 100 were further selected. The simulation was conducted for three (3) different cases using varying true parameter values. The selected true parameter values are $\alpha=0.5, \beta=0.5, \gamma=0.5$, and $\theta=0.5 ; \alpha=1, \beta=1$, $\gamma=1$, and $\theta=1$; and $\alpha=2, \beta=2, \gamma=2$, and $\theta=2$ for the first, second, and third cases, respectively.

The MLE of the true parameters were obtained including the Bias and the Root Mean Square Error (RMSE). The result for the simulation studies is as shown in Tables 1, 2, and 3.

Remark 3. It can be deduced from Tables 1,2 , and 3 that the root mean square error (RMSE) reduces for all the selected parameter values as the sample size increases. Also, the bias posed by the estimates is closer to the true parameter values and the absolute bias reduces as the sample size increases. Hence, as sample size increases, the estimates tend towards (or approaches) the true parameter values.

\section{Application}

To demonstrate the potentials of the GoLom distribution,

\begin{tabular}{|c|c|c|c|c|}
\hline$n$ & Parameters & Means & Bias & RMSE \\
\hline \multirow{4}{*}{25} & $\alpha=0.5$ & 0.4849 & -0.0151 & 0.1974 \\
\hline & $\beta=0.5$ & 0.5379 & 0.0379 & 0.2012 \\
\hline & $\gamma=0.5$ & 0.5469 & 0.0469 & 0.1134 \\
\hline & $\theta=0.5$ & 0.5552 & 0.0552 & 0.3301 \\
\hline \multirow{4}{*}{50} & $\alpha=0.5$ & 0.4913 & -0.0087 & 0.1763 \\
\hline & $\beta=0.5$ & 0.5204 & 0.0204 & 0.1655 \\
\hline & $\gamma=0.5$ & 0.5284 & 0.0284 & 0.0861 \\
\hline & $\theta=0.5$ & 0.5544 & 0.0544 & 0.2889 \\
\hline \multirow{4}{*}{100} & $\alpha=0.5$ & 0.5080 & 0.0080 & 0.1603 \\
\hline & $\beta=0.5$ & 0.5131 & 0.0131 & 0.1334 \\
\hline & $\gamma=0.5$ & 0.5100 & 0.0100 & 0.0658 \\
\hline & $\theta=0.5$ & 0.5394 & 0.0394 & 0.2355 \\
\hline
\end{tabular}
a comparison was made using the GoLom distribution and some other compound distributions like Beta Lomax

\begin{tabular}{|c|c|c|c|c|}
\hline$n$ & Parameters & Means & Bias & RMSE \\
\hline \multirow{4}{*}{25} & $\alpha=1.0$ & 0.8897 & -0.1103 & 0.2947 \\
\hline & $\beta=1.0$ & 1.0938 & 0.0938 & 0.3420 \\
\hline & $\gamma=1.0$ & 1.1110 & 0.1110 & 0.2602 \\
\hline & $\theta=1.0$ & 1.0330 & 0.0330 & 0.2509 \\
\hline \multirow{4}{*}{50} & $\alpha=1.0$ & 0.9189 & -0.0811 & 0.2172 \\
\hline & $\beta=1.0$ & 1.0592 & 0.0592 & 0.2449 \\
\hline & $\gamma=1.0$ & 1.0545 & 0.0545 & 0.1671 \\
\hline & $\theta=1.0$ & 1.0397 & 0.0397 & 0.2082 \\
\hline \multirow{4}{*}{100} & $\alpha=1.0$ & 0.9490 & -0.0510 & 0.1593 \\
\hline & $\beta=1.0$ & 1.0465 & 0.0465 & 0.1733 \\
\hline & $\gamma=1.0$ & 1.0096 & 0.0096 & 0.1137 \\
\hline & $\theta=1.0$ & 1.0470 & 0.0470 & 0.1514 \\
\hline
\end{tabular}
distribution, Weibull Lomax distribution, and Kumaraswamy
TABLE 1: Simulation study at $\alpha=0.5, \beta=0.5, \gamma=0.5$, and $\theta=0.5$.

\begin{tabular}{|c|c|c|c|c|}
\hline$n$ & Parameters & Means & Bias & RMSE \\
\hline \multirow{4}{*}{25} & $\alpha=2.0$ & 1.8415 & -0.1585 & 0.7105 \\
\hline & $\beta=2.0$ & 2.1605 & 0.1605 & 0.7190 \\
\hline & $\gamma=2.0$ & 2.2196 & 0.2196 & 0.4790 \\
\hline & $\theta=2.0$ & 2.0191 & 0.0191 & 0.3825 \\
\hline \multirow{4}{*}{50} & $\alpha=2.0$ & 1.9171 & -0.0829 & 0.5363 \\
\hline & $\beta=2.0$ & 2.1175 & 0.1175 & 0.5653 \\
\hline & $\gamma=2.0$ & 2.1155 & 0.1155 & 0.3550 \\
\hline & $\theta=2.0$ & 2.0055 & 0.0055 & 0.3407 \\
\hline \multirow{4}{*}{100} & $\alpha=2.0$ & 1.9881 & -0.0119 & 0.3892 \\
\hline & $\beta=2.0$ & 2.0890 & 0.0890 & 0.4110 \\
\hline & $\gamma=2.0$ & 2.0507 & 0.0507 & 0.2777 \\
\hline & $\theta=2.0$ & 1.9862 & -0.0138 & 0.2962 \\
\hline
\end{tabular}

TABLE 2: Simulation study at $\alpha=1.0, \beta=1.0, \gamma=1.0$, and $\theta=1.0$.

TABLE 3: Simulation study at $\alpha=2.0, \beta=2.0, \gamma=2.0$, and $\theta=2.0$.

Lomax distribution. The following criteria were used to select the distribution with the best fit: Negative Log-Likelihood (-LL) value, Akaike Information Criteria (AIC), Bayesian Information Criteria (BIC), Consistent Akaike Information 
TABle 4: Performance rating of the GoLom distribution.

\begin{tabular}{|c|c|c|c|c|c|c|}
\hline Distributions & Estimates & $-\mathrm{LL}$ & AIC & CAIC & $\mathrm{BIC}$ & HQIC \\
\hline Gompertz Lomax & $\begin{array}{l}\widehat{\alpha}=0.0046 \\
\widehat{\beta}=8.1791 \\
\widehat{\gamma}=1.5158 \\
\widehat{\theta}=0.5069\end{array}$ & 14.5027 & 37.0055 & 37.6951 & 45.5780 & 40.3771 \\
\hline Weibull Lomax & $\begin{array}{l}\widehat{\alpha}=6.0947 \\
\widehat{\beta}=0.1069 \\
\widehat{a}=1.0629 \\
\widehat{b}=0.0649\end{array}$ & 15.3399 & 38.6798 & 39.3695 & 47.2524 & 42.0514 \\
\hline Beta Lomax & $\begin{array}{c}\widehat{\alpha}=18.1737 \\
\widehat{\beta}=26.7645 \\
\widehat{a}=10.8769 \\
\widehat{b}=0.0329\end{array}$ & 24.4034 & 56.8068 & 57.4964 & 65.3793 & 60.1784 \\
\hline Kumaraswamy Lomax & $\begin{array}{c}\widehat{\alpha}=9.8352 \\
\widehat{\beta}=45.3107 \\
\widehat{a}=15.1182 \\
\widehat{b}=0.0483\end{array}$ & 18.1027 & 44.2055 & 44.8951 & 52.7779 & 47.5771 \\
\hline
\end{tabular}

TABLE 5: Table of test statistic.

\begin{tabular}{lccc}
\hline Distributions & KS & $A$ & $p$ value \\
\hline Gompertz Lomax & 0.1542 & 0.9462 & 0.0998 \\
Weibull Lomax & 0.1517 & 1.3315 & 0.1100 \\
Beta Lomax & 0.2182 & 3.1986 & 0.0049 \\
Kumaraswamy Lomax & 0.1854 & 1.9915 & 0.0263 \\
\hline
\end{tabular}

Criteria (CAIC), and Hannan and Quinn Information Criteria (HQIC). The value for the Kolmogorov Smirnov (KS) statistic, Anderson Darling (A) statistic, and the $p$ value are also provided.

The data relating to the strengths of $1.5 \mathrm{~cm}$ glass fibres which was obtained by workers at the UK National Physical Laboratory was used. The data has previously been used by Smith and Naylor [17], Bourguinon et al. [18], and Merovci et al. [19]. The observations are as follows:

$0.55,0.74,0.77,0.81,0.84,1.24,0.93,1.04,1.11,1.13,1.30$, $1.25,1.27,1.28,1.29,1.48,1.36,1.39,1.42,1.48,1.51,1.49,1.49$, $1.50,1.50,1.55,1.52,1.53,1.54,1.55,1.61,1.58,1.59,1.60,1.61$, $1.63,1.61,1.61,1.62,1.62,1.67,1.64,1.66,1.66,1.66,1.70,1.68$, $1.68,1.69,1.70,1.78,1.73,1.76,1.76,1.77,1.89,1.81,1.82,1.84$, $1.84,2.00,2.01,2.24$.

The performances of the GoLom distribution with the other competing distributions are shown in Table 4.

Remark 4. The distribution that corresponds to the lowest -LL, AIC, CAIC, BIC, and HQIC is judged to be the best out of the competing distributions. With this regard, the competing distributions can be ranked in the following order (best to the least): Gompertz Lomax distribution, Weibull Lomax distribution, Kumaraswamy Lomax distribution, and Beta Lomax distribution.

The values for the Kolmogorov Smirnov statistic, Anderson Darling statistic, and the $p$ value are as shown in Table 5.

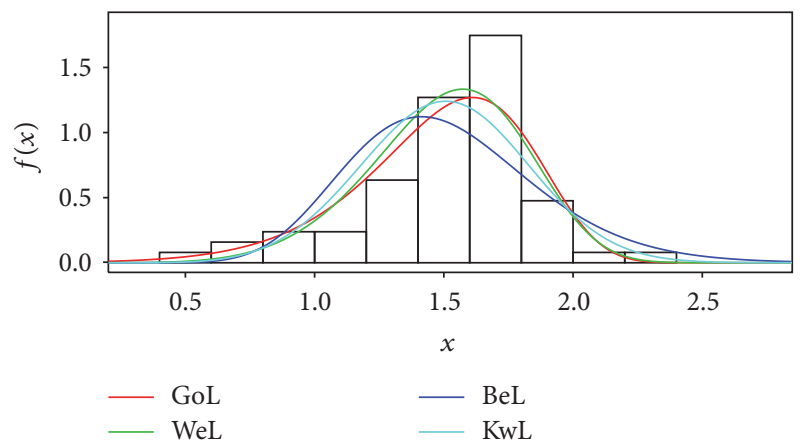

FIgURE 4: Plot showing the competing distributions with the empirical histogram of the observed data.

A plot showing all the competing distributions against the empirical histogram of the observed data is as shown in Figure 4.

A plot for the empirical cdf of the competing distributions with the empirical cdf of the observed data is as shown in Figure 5.

The plots in Figures 4 and 5 affirm the results of the analysis that the Gompertz Lomax distribution is more suitable for the data than the other competing distributions.

\section{Conclusion}

The Gompertz Lomax distribution has been successfully derived; expressions for its basic statistical properties which include the reliability function, hazard function, odds function, reversed hazard function, and quantile, median, and distribution of order statistics have been successfully established. The shape of the distribution could be decreasing or inverted bathtub (depending on the value of the parameters). Meanwhile, the shape of its hazard function could be constant, increasing, or decreasing (depending on the value of the 


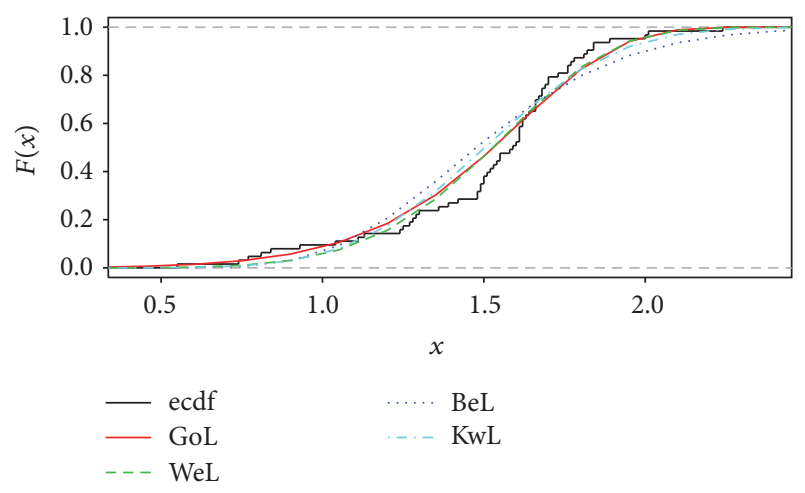

FIGURE 5: Plot for the empirical cdf of the competing distributions.

parameters). The simulation study that was conducted shows that the parameters of the Gompertz Lomax distribution are stable; though values for biasedness were generated, these values are small, indicating that the maximum likelihood estimates of the GoLom distribution are not too far from the true parameter values; the absolute bias and the root mean square values also decreases as the sample size increases. An application to a real life data shows that the Gompertz Lomax distribution is a strong and better competitor for the Weibull Lomax distribution, Beta Lomax distribution, and Kumaraswamy Lomax distribution.

\section{Conflicts of Interest}

The authors declare that there are no conflicts of interest.

\section{Acknowledgments}

The authors are grateful to Covenant University for providing funding and an enabling environment for this research.

\section{References}

[1] B. Al-Zahrani and M. Al-Sobhi, "On parameters estimation of Lomax distribution under general progressive censoring," Journal of Quality and Reliability Engineering, vol. 2013, Article ID 431541, 7 pages, 2013.

[2] K. S. Lomax, "Business failures: another example of the analysis of failure data," Journal of the American Statistical Association, vol. 49, no. 268, pp. 847-852, 1954.

[3] A. Hassan and A. Al-Ghamdi, "Optimum step stress accelerated life testing for Lomax distribution," Journal of Applied Sciences Research, vol. 5, pp. 2153-2164, 2009.

[4] N. M. Kilany, "Weighted Lomax distribution," SpringerPlus, vol. 5, no. 1, article no. 1862, 2016.

[5] A. H. El-Bassiouny, N. F. Abdo, and H. S. Shahen, "Exponential lomax distribution," International Journal of Computer Applications, vol. 121, no. 13, pp. 24-29, 2015.

[6] H. M. Salem, "The exponentiated lomax distribution: different estimation methods," American Journal of Applied Mathematics and Statistics, vol. 2, no. 6, pp. 364-368, 2014.

[7] G. M. Cordeiro, E. M. M. Ortega, and B. V. Popović, "The gamma-Lomax distribution," Journal of Statistical Computation and Simulation, vol. 85, no. 2, pp. 305-319, 2015.
[8] S. K. Ashour and M. A. Eltehiwy, "Transmuted lomax distribution," American Journal of Applied Mathematics and Statistics, vol. 1, no. 6, pp. 121-127, 2013.

[9] B. Al-Zahrani and H. Sagor, "The Poisson-Lomax distribution," Revista Colombiana de Estadística, vol. 37, no. 1, pp. 225-245, 2014.

[10] A. J. Lemonte and G. M. Cordeiro, "An extended Lomax distribution," A Journal of Theoretical and Applied Statistics, vol. 47, no. 4, pp. 800-816, 2013.

[11] M. H. Tahir, G. M. Cordeiro, M. Mansoor, and M. Zubair, "The weibull-lomax distribution: properties and applications," Hacettepe Journal of Mathematics and Statistics, vol. 44, no. 2, pp. 461-480, 2015.

[12] E.-H. A. Rady, W. A. Hassanein, and T. A. Elhaddad, "The power Lomax distribution with an application to bladder cancer data," SpringerPlus, vol. 5, 1838 pages, 2016.

[13] E. A. Owoloko, P. E. Oguntunde, and A. O. Adejumo, "Performance rating of the transmuted exponential distribution: an analytical approach," SpringerPlus, vol. 4, 818 pages, 2015.

[14] P. E. Oguntunde, A. O. Adejumo, H. I. Okagbue, and M. K. Rastogi, "Statistical properties and applications of a new lindley exponential distribution," Gazi University Journal of Science, vol. 29, no. 4, pp. 831-838, 2016.

[15] G. M. Cordeiro, M. Alizadeh, A. D. C. Nascimento, and M. Rasekhi, "The exponentiated Gompertz generated family of distributions: properties and applications," Chilean Journal of Statistics, vol. 7, no. 2, pp. 29-50, 2016.

[16] M. Alizadeh, G. M. Cordeiro, L. G. Bastos Pinho, and I. Ghosh, "The Gompertz-G Family of distributions," Journal of Statistical Theory and Practice, vol. 11, no. 1, pp. 179-207, 2017.

[17] R. L. Smith and J. C. Naylor, "A comparison of maximum likelihood and bayesian estimators for the three-parameter weibull distribution," Applied Statistics, vol. 36, pp. 258-369, 1987.

[18] M. Bourguignon, R. B. Silva, and G. M. Cordeiro, "The weibullG family of probability distributions," Journal of Data Science, vol. 12, pp. 53-68, 2014.

[19] F. Merovci, M. A. Khaleel, N. A. Ibrahim, and M. Shitan, "The beta type X distribution: properties with application," SpringerPlus, vol. 5, 697 pages, 2016. 


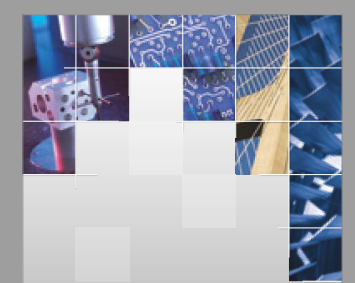

\section{Enfincering}
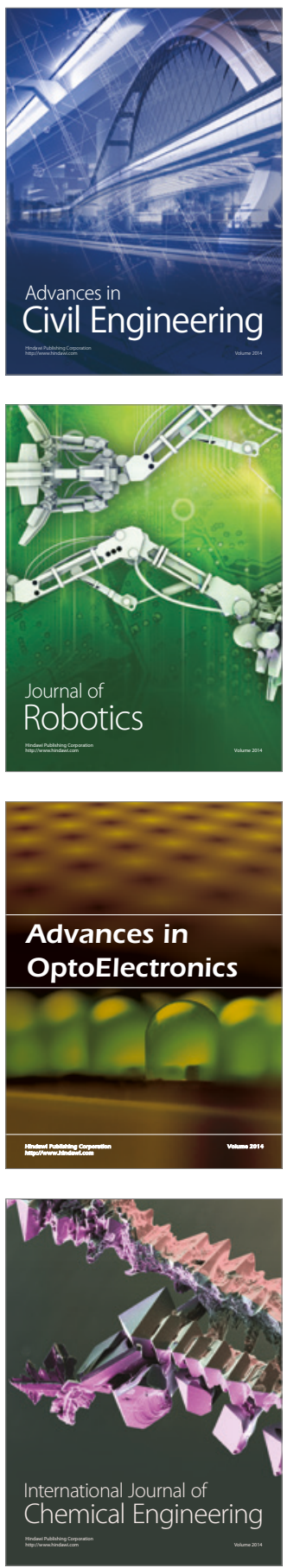

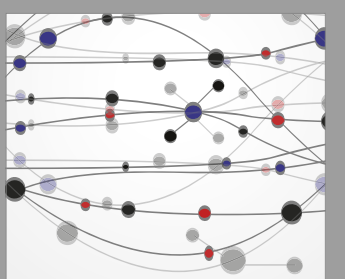

The Scientific World Journal

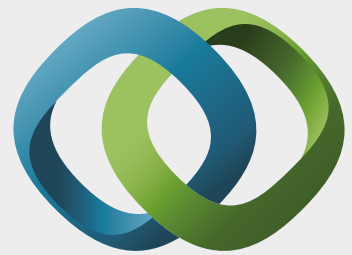

\section{Hindawi}

Submit your manuscripts at

https://www.hindawi.com
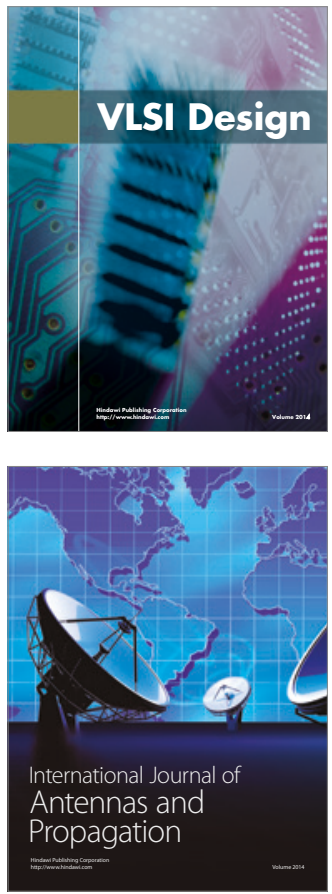

\section{Rotating}

Machinery
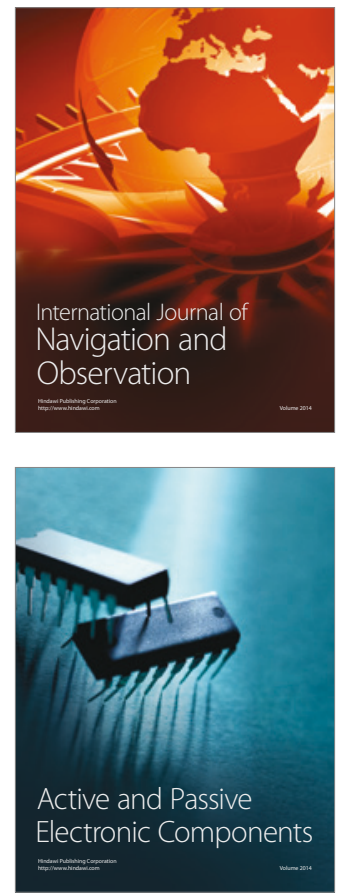
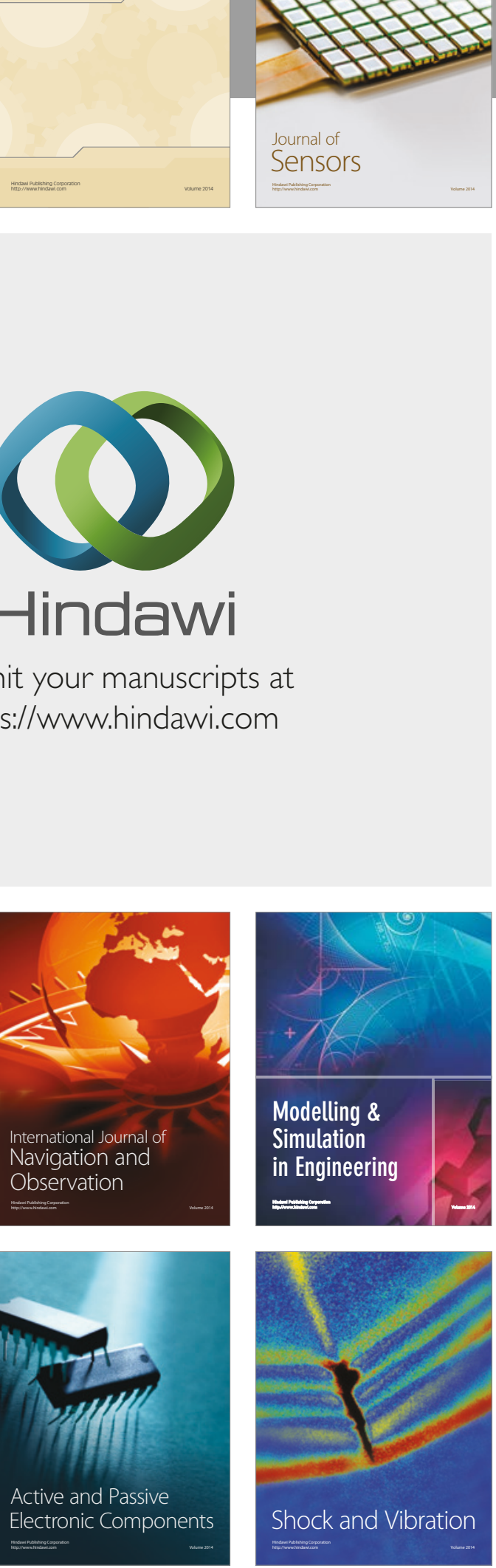
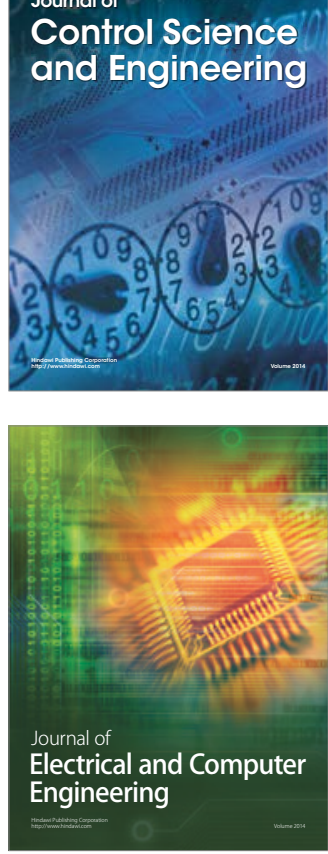

Distributed

Journal of

Control Science

and Engineering
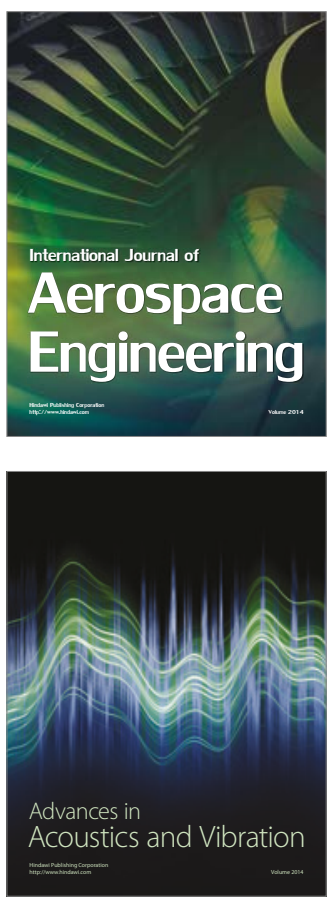

Sensor Networks 